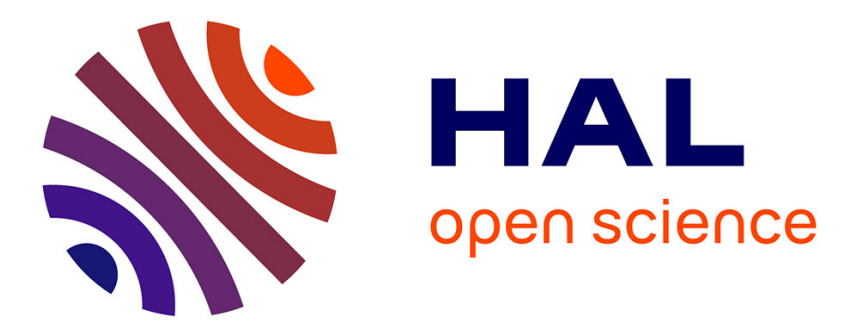

\title{
Linear temperature dependence of the constant volume resistivity of TTF-TCNQ
}

\author{
R.H. Friend, M. Miljak, D. Jérome, D.L. Decker, D. Debray
}

\section{To cite this version:}

R.H. Friend, M. Miljak, D. Jérome, D.L. Decker, D. Debray. Linear temperature dependence of the constant volume resistivity of TTF-TCNQ. Journal de Physique Lettres, 1978, 39 (9), pp.134-138. 10.1051/jphyslet:01978003909013400 . jpa-00231461

\section{HAL Id: jpa-00231461 https://hal.science/jpa-00231461}

Submitted on 1 Jan 1978

HAL is a multi-disciplinary open access archive for the deposit and dissemination of scientific research documents, whether they are published or not. The documents may come from teaching and research institutions in France or abroad, or from public or private research centers.
L'archive ouverte pluridisciplinaire HAL, est destinée au dépôt et à la diffusion de documents scientifiques de niveau recherche, publiés ou non, émanant des établissements d'enseignement et de recherche français ou étrangers, des laboratoires publics ou privés. 


\author{
Classification \\ Physics Abstracts \\ $72.15-72.15 \mathrm{~N}-72.80 \mathrm{~L}$
}

\title{
LINEAR TEMPERATURE DEPENDENCE OF THE CONSTANT VOLUME RESISTIVITY OF TTF-TCNQ $\left(^{*}\right)$
}

\author{
R. H. FRIEND $(* *)$, M. MILJAK $(* * *)$, D. JÉROME \\ Laboratoire de Physique des Solides, Université Paris-Sud, 91405 Orsay, France \\ D. L. DECKER (****) and D. DEBRAY \\ Laboratoire Léon Brillouin, CEN de Saclay, 91190 Gif sur Yvette, France
}

(Reçu le 15 février 1978, accepté le 14 mars 1978)

\begin{abstract}
Résumé. - Les expériences de diffraction de neutrons sous haute pression démontrent que les inclinaisons des molécules de TTF et de TCNQ par rapport à l'axe d'empilement ne varient pas sous pression. Ce résultat est utilisé pour obtenir la variation en température de la conductivité longitudinale à volume constant.

La loi de variation $T^{2,3}$ sous pression constante devient alors une loi linéaire en température.

Abstract. - High pressure neutron diffraction experiments show that the angles of tilt of the TCNQ and TTF molecules to the stacking axis do not change under pressure. This result, together with measurements of the pressure dependence of the conductivity as a function of temperature, is used to derive the constant volume stacking axis resistivity of TTF-TCNQ. The temperature dependence is strongly reduced from the $T^{2.3}$ law at constant pressure, and is better characterized by a linear $T$ law.
\end{abstract}

1. Introduction. - The anomalous temperature dependence of the conductivity of TTF-TCNQ in the metallic regime $(T>53 \mathrm{~K})$ remains controversial. The strong temperature dependence is characterized by a power law of the form $\rho=\rho_{0}+A T^{\lambda}$ with $\lambda>2.3$ for good quality samples $[1,2]$. Besides the collective electron-phonon current carrying mechanism suggested by the Pennsylvannia group [3], which has been criticized by Thomas et al. [4], scattering mechanisms proposed for single particle conduction include electron-electron [5], electron-magnon [6], electron-molecular vibration [7] and electron-libron [8] collisions. Phonon induced localization has also been considered [9-11].

Recently, Cooper [12] has drawn attention to the strong pressure dependence of the metallic regime resistivity, which has not generally been adequately taken into account in the proposed scattering models.

$\left({ }^{*}\right)$ Work supported in part by DGRST contract $n^{\circ}$ 75-7-0820. U.K.

(**) Permanent address : Cavendish Laboratory, Cambridge,

$(* * *)$ Permanent address : Institute of physics of the University, P.O. Box 304, Zagreb, Yugoslavia.

$(* * *)$ On leave from : Brigham Young University, Provo, Utah, 84601, U.S.A.
In particular, Cooper has demonstrated that, when corrected for the effect of thermal expansion, the constant b axis resistivity of TTF-TCNQ has a temperature dependence closer to linear than to a $T^{2.3}$ power law. We have obtained new data, of both the high pressure structure and the high pressure resistivity, and use this to derive more accurately the temperature dependence of the constant volume resistivity. We find that it is satisfactorily characterized by a linear power law, particularly above $150 \mathrm{~K}$. We present results for samples which showed ratios of $\sigma($ peak $) / \sigma(300 \mathrm{~K})$ of 15 and 25 , and do not find that this result depends significantly on sample quality.

2. Neutron diffraction study of the high pressure structure of TTF-TCNQ. - We have looked for changes in the angles of tilt of the TTF and TCNQ molecules with respect to the stacking axis, under pressure, by analysing intensity changes in the Bragg peaks measured in high pressure neutron diffraction experiments on deuterated TTF-TCNQ. Experimental data were taken from experiments previously analysed to determine the compressibility [13], together with similar experiments to follow Bragg peaks not previously measured. Best determinations were made from comparison of the intensity ratios of peaks which 
are relatively insensitive to changes in lattice parameter, but change rapidly with changes in either or both of the tilt angles.

Assuming, as a worst case, that all the $c$ axis compressibility derives from increases in the tilt angles, by $20 \mathrm{kbar}$ the TCNQ angle (340 at 1 bar [14]) will have increased by $3^{\circ}$, and the TTF angle $\left(24.5^{\circ}\right.$ at 1 bar [14]) by $4^{\circ}$. We have calculated intensity changes for TCNQ/TTF tilt angle increases in the ratio $3 / 4$, and also for changes in only one stack. Intensity changes are approximately linear with change in angle.

For the 112 and 013 lines (Fig. 1) the measured ratio is $0.263 \pm 0.011$ at 1 bar and $0.266 \pm 0.013$ at $15 \mathrm{kbar}$; the change in the ratio with pressure is thus $1.01 \pm 0.06$. Calculated changes are : 1.01 from the application of $15 \mathrm{kbar}$ without tilt angle changes, 0.60 from $4^{\circ} \mathrm{TTF}$ and $3^{\circ} \mathrm{TCNQ}$ tilt, 0.75 from $4^{\circ} \mathrm{TTF}$ tilt only, and 0.84 from $3^{\circ}$ TCNQ tilt only. Thus if both tilt angles change together,

and

$$
\Delta \theta(\mathrm{TCNQ})=0.0 \pm 0.4^{\circ}
$$

$$
\Delta \theta(\mathrm{TTF})=0.0 \pm 0.6^{\circ}
$$

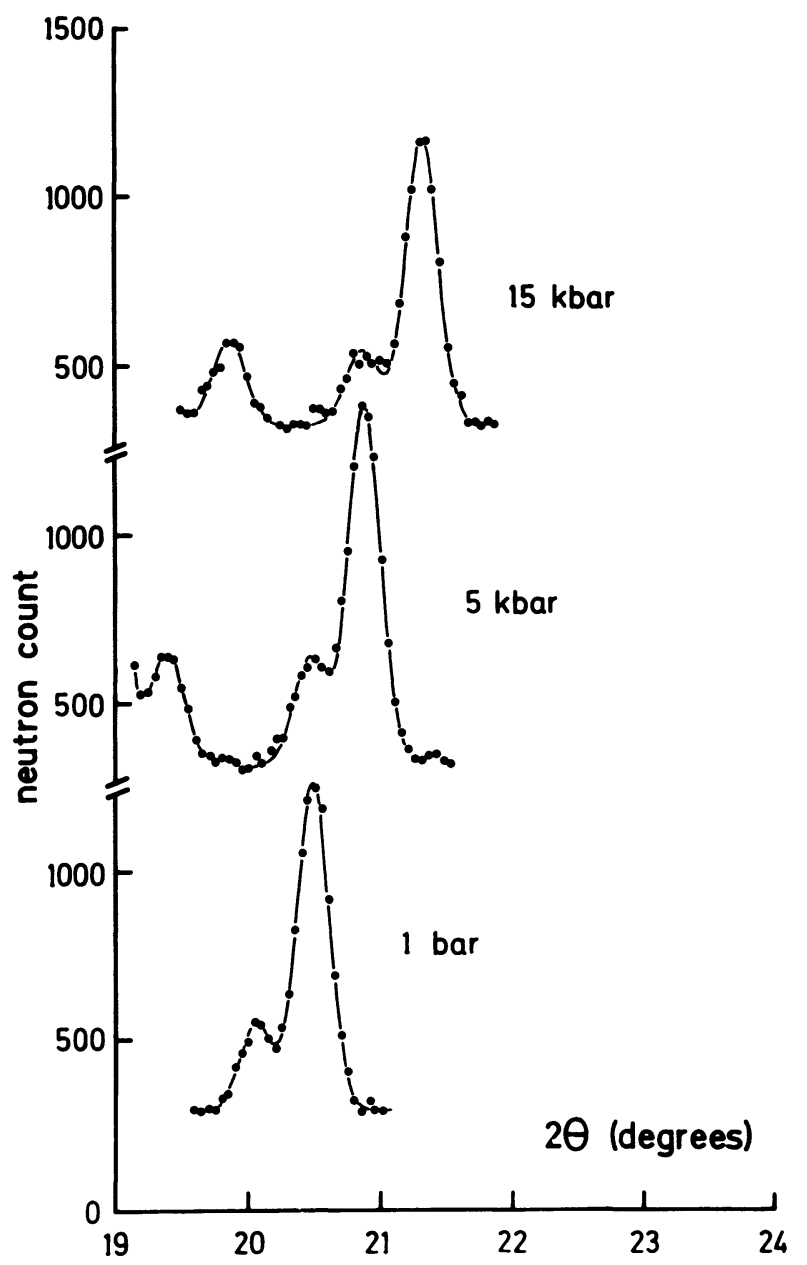

Fig. 1. - Pressure dependence of the, from left to right, $(11 \overline{2})$ (not shown at 1 bar), 112 and 013 Bragg angles of a deuterated sample of TTF-TCNQ at room temperature $(\lambda=1.14 \AA)$.
In the unphysical situation of only one stack changing, the limits are $\Delta \theta<1.1^{\circ}$ for TCNQ and $\Delta \theta<0.9^{\circ}$ for TTF. Comparison of the $11 \overline{2}$ line, also seen in figure 1, but which is only separated from an alumina cell peak above $5 \mathrm{kbar}$, to the 013 line, gives $\Delta \theta$ (TTF) $<0.7^{\circ}$ and $\Delta \theta(\mathrm{TCNQ})<0.5^{\circ}$ between 5 and $15 \mathrm{kbar}$. No other lines investigated showed any evidence for tilt angle changes, and we conclude that neither tilt angle changes by more than $\sim 0.6^{\circ}$ in $15 \mathrm{kbar}$.

This estimate is consistent with the small differences in $a$ and $c$ axis compressibilities [13]. Only the $c$ axis is affected by tilt angle changes, and if the slightly larger $c$ axis compressibility is attributed to such an effect, the increases in tilt angles are less than $0.5^{\circ}$ at $20 \mathrm{kbar}$.

This independence of the tilt angles with pressure is surprising. The tilt angle is generally considered to be determined by the most favourable orbital overlap between molecules in a stack (in the case of TCNQ, the stacking of the carbon-carbon double bonds directly above the 6 membered rings [14]). With decreasing $b$ axis under pressure, the angle of tilt would have to increase to preserve the same relative displacement between molecules on the stack; at $20 \mathrm{kbar}$ by $2^{\circ}$ for the TCNQ stack and by $1.5^{\circ}$ for the TTF stack. However the tilt angles are also found to be temperature independent [15], and both donor and acceptor tilt angles keep the same values in different TCNQ salts (TSF-TCNQ [16], HMTTF-TCNQ [17], HMTSF-TCNQ [18]), in spite of differences in the $b$ axis parameter.

3. Pressure dependence of the metallic regime conductivity of TTF-TCNQ. - The linear pressure dependence of the room temperature $b$ axis conductivity of TTF-TCNQ is shown in figure 2. Normal samples with $\sigma($ peak $) / \sigma(300 \mathrm{~K}) \sim 15$ were used, and a mixture of isopentane and isoamyl alcohol was employed as the pressure transmitting medium. The

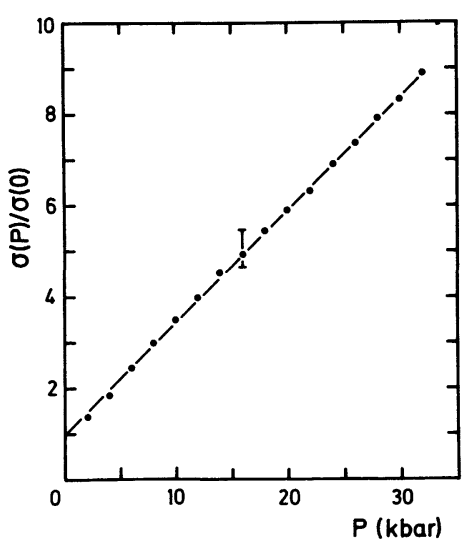

Fig. 2. - Pressure dependence of the stacking axis conductivity of TTF-TCNQ at room temperature, for a normal sample with $\sigma($ peak $) / \sigma(300 \mathrm{~K}) \sim 15$. Error bar indicates range of variation between samples, those with higher $\sigma($ peak $) / \sigma(300 \mathrm{~K})$ showing larger values of $\mathrm{d} \sigma / \mathrm{d} P$. 
data presented in this figure extend the pressure range of previous investigations [19-21] up to $32 \mathrm{kbar}$ [22].

We have made new measurements of the stacking axis resistivity under pressure to obtain accurate values of the pressure dependence of the resistivity as a function of temperature, particularly at low pressures. The crystals used for these experiments were ${ }^{15} \mathrm{~N}$ substituted samples, which have previously been found to show sharp M-I transitions [23]. High pressure helium gas equipment with automatic pressure regulation to within \pm 20 bars was used [24], and all measurements in the metallic regime were above the helium freezing point. The procedure used to measure $\mathrm{d} \sigma / \mathrm{d} P$ as a function of temperature was to cool the sample under pressure, release pressure at low temperature, and warm at ambient pressure. $\sigma$ (peak)/ $\sigma(300 \mathrm{~K})$, after pressure release, was between 15 and 25 . Figure 3 shows data for a sample with a ratio of 25 . Unnested ratios were always more than 20 at $\sigma$ (peak) [25]. Also shown in figure 3 are representative plots of $(\sigma(P)-\sigma(0)) / P \sigma(0)$. This quantity is independent of pressure at room temperature (i.e. $\sigma$ increases linearly

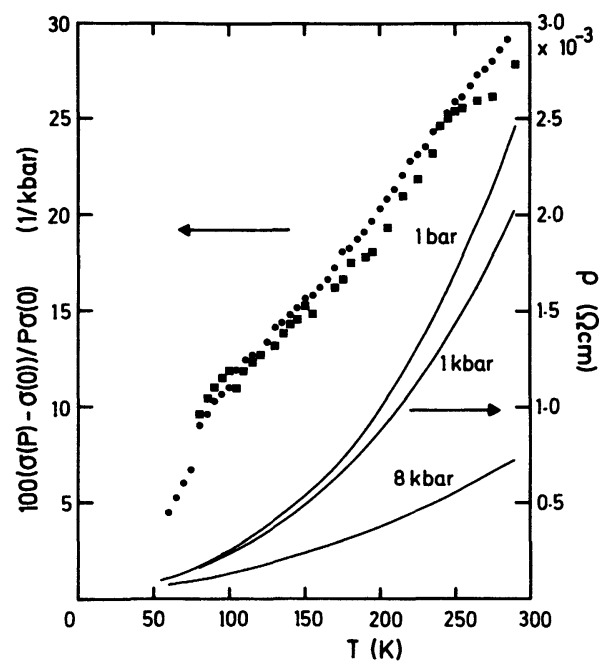

FIG. 3. - Right hand scale : resistivity versus temperature at constant pressure for TTF-TCNQ \# 7. ( $\rho(300 \mathrm{~K}, 1 \mathrm{bar})$ is normalized to $\left.2.5 \times 10^{-3} \Omega \mathrm{cm}\right)$. Left hand scale : $(\sigma(P)-\sigma(1$ bar $)) /$ $P \sigma(1$ bar) versus temperature. $\bullet$ data for \# 7 between 1 bar and $8 \mathrm{kbar}$ data for \# 8 between 1 bar and $1 \mathrm{kbar}$.

with pressure, figure 2), and is conveniently, pressure independent over the whole temperature range. For the data reduction in the following section we approximated the temperature dependence of this quantity by a simple linear law,

$(\sigma(P, T)-\sigma(0, T)) / P \sigma(0, T)=(T / 300) \times 28 \% / \mathrm{kbar}$.

We did notice that for samples with very poor peak conductivities, the pressure dependence below $100 \mathrm{~K}$ was considerably higher than as shown in figure 3 . This may result from a pressure dependent anisotropy in the region of the transitions if the poor conductivity results from a transverse component of the conductivity being picked up in the measurement.

4. Derivation of constant volume resistivity. $X$-ray measurements of the thermal expansion show increases of $1.1 \%, 2.3 \%$ and $0.8 \%$ in the $a, b$ and $c$ axes between 60 and $295 \mathrm{~K}$ [16], and high pressure neutron diffraction measurements give the room temperature compressibilities as $0.27 \%, 0.47 \%$ and $0.32 \% / \mathrm{kbar}$ for the $a, b$ and $c$ axes [13]. Whilst there is no simple correspondence between the thermal expansion and compressibility, we follow Cooper [12] who chose to keep the stacking axis cell length, $b$ to its value at $60 \mathrm{~K}$, since most of the thermal expansion and compressibility is in this direction.

Since the anisotropy in compressibility is smaller than that in the thermal expansion, correcting at a high temperature by applying pressure to reduce the $b$ axis will result in $a$ and $c$ axes smaller than the ambient pressure low temperature values. This will, increase slightly the values of the transverse transfer integral, $t_{\perp}$. However the measured resistivity anisotropy is almost pressure independent [26] and this has been understood in terms of diffuse motion of carriers between stacks with a weakly pressure dependent $t_{\perp}$ [27]. A more serious objection is that the reduction in the $c$ axis could come about from an increase in the tilt angles of the two stacks with respect to the $b$ axis. This would increase the overlap between molecules in each stack, in the same way as a reduction in the $b$ axis, and upset the thermal expansion-compressibility $b$ axis equivalence. The experimentally measured independence of the tilt angles with pressure removes this objection.

Direct evidence that the $b$ axis determines the pressure behaviour of the conductivity comes from recent measurements of the effect of uniaxial strain on TTF-TCNQ [28], which show that strain applied along the $b$ axis has an order of magnitude larger effect on the $b$ axis conductivity than strain along the $a$ axis.

We have included the pressure dependence of the $b$ axis compressibility by fitting the $b$ axis compressive axial strain versus pressure data [13] to a quartic in $\Delta b / b$. To obtain the compressibility below room temperature we have used the temperature dependence of the longitudinal $b$ axis sound velocity, from the LA phonon branch determined by inelastic neutron diffraction [29], to scale the room temperature value. Including corrections for the density change with thermal expansion, the calculated effect is to decrease the compressibility at $60 \mathrm{~K}$ by $8 \%$ from its room temperature value. The values of $b(T)$ used [30] were obtained from the data of references [16] and [31], with $\Delta b / b$ between $300 \mathrm{~K}$ and $60 \mathrm{~K}$ equal to $2.34 \%$.

Constant $b$ axis resistivity versus temperature curves shown in figure 4 were obtained by calculating at each temperature the pressure required to bring the $b$ lattice parameter to its value at $60 \mathrm{~K}$, and correcting the measured value of the resistivity to its value at that 
pressure. Thus a reduction in $b$ by $2.34 \%$ at $300 \mathrm{~K}$ requires a pressure of $5 \mathrm{kbar}$, increasing the conductivity to 2.4 times its ambient pressure value. It is evident from figures 3 and 4 that the $\left.\rho(T)\right|_{\text {constant } P}$ and $\left.\rho(T)\right|_{\text {constant } b}$ curves are quite different; that the temperature dependence of $\left.\rho(T)\right|_{\text {constant } b}$ is much weaker than $\left.\rho(T)\right|_{\text {constant } P}$ and is quite close to a linear $T$ law.

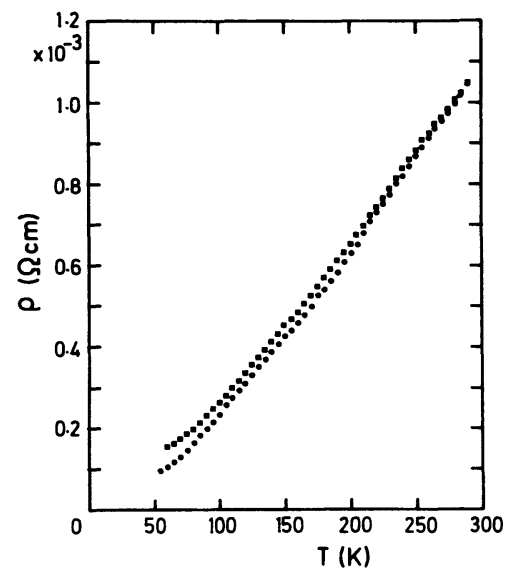

FIG. 4. - Resistivity versus temperature for TTF-TCNQ with $b$ lattice parameter constant at its $60 \mathrm{~K}$ value. - data for \# 7 $(\sigma($ peak $) / \sigma(300 \mathrm{~K})$ at 1 bar $=25), \square$ data for \# $8(\sigma($ peak $) / \sigma(300 \mathrm{~K})$ at $1 \mathrm{bar}=15) . \rho(300 \mathrm{~K}, 1 \mathrm{bar})$ is normalized to $2.5 \times 10^{-3} \Omega \mathrm{cm}$ for both samples.

We have performed curve fits to $\rho=\rho_{0}+A T^{\lambda}$, minimizing $\sum_{i}\left(\rho-\rho_{i}\right)^{2}$, where $\rho_{i}$ are the experimental points at $5 \mathrm{~K}$ intervals. For sample \# 7, seen in figures 3 and 4 , and which showed $\sigma($ peak $) / \sigma(300 \mathrm{~K})=25$, we find that at atmospheric pressure $\lambda=2.58$ when the fit is between 60 and $290 \mathrm{~K}$. Dividing the range into $60-150 \mathrm{~K}$ and $150-290 \mathrm{~K}$, we find exponents of 1.84 and 2.66 respectively. When corrected to constant $b$ axis, the simple power law fit does not cover the whole temperature range so satisfactorily; thus we find $\lambda=1.29$ for a fit from 60 to $300 \mathrm{~K}$, but exponents of 1.00 and 1.22 for $150-290 \mathrm{~K}$ and $60-150 \mathrm{~K}$ fits. We attach no particular significance to these values, and give them merely to aid comparison between figures 3 and 4 .

The second sample shown in figure $4, \# 8$, showed a peak ratio of 15 . We note that the constant $b$ axis resistivity deviates above that of \# 7 right from $300 \mathrm{~K}$ (where the two are normalized to the same value), and that differences in peak ratio do not lead to large variations in $\mathrm{d} \ln \sigma / \mathrm{d} \ln T$ in any particular temperature regime, although there is a tendency for $\rho$ to saturate below $100 \mathrm{~K}$ in the poorer sample (values of $\lambda$ for \# 8 are 1.30 for $60-290 \mathrm{~K}, 1.00$ for $150-290 \mathrm{~K}$ and 1.57 for $60-150 \mathrm{~K}$ ). We do not consider that the form of the curves in figure 4 is significantly dependent on sample purity/peak ratio.
We have assessed the sensitivity of the constant volume plots to the various input parameters. Using a pressure independent compressibility, or using the data points in figure 3 rather than the linear approximation to $(\sigma(P)-\sigma(0)) / P \sigma(0)$ made no discernible difference. Using the temperature dependence of the elongational mode $b$ axis sound velocity [32] to scale the compressibility at low temperatures decreases the low temperature compressibility faster than does the inelastic neutron data; this has the effect of increasing the exponents in the power law fits by $\sim 0.1$.

Although we attach no particular significance to the detailed shapes of the constant volume resistivity curves we have derived, we consider that the weak, quasi linear temperature dependence is well established. This result is not much affected by sample quality, and we point out that we have presented sufficient information on the pressure dependence of the resistivity (in figure 3 ) for the same data reduction to be performed on any other $\rho(T)$ at atmospheric pressure data.

As emphasized by Cooper [12], a successful model for the scattering mechanism that determines the resistivity must be able to account for the strong pressure dependence, as well as the quasi linear temperature dependence. We comment here on those models in which the pressure dependence has been considered.

Conwell [7] requires a pressure dependent impurity resistivity as well as the molecular vibration-electron collision limited mobility, implying a larger value of $\mathrm{d} \ln \sigma / \mathrm{d} P$ for poorer quality samples. We have found only a small sample dependence and larger values of $\mathrm{d} \ln \sigma / \mathrm{d} P$ in samples with higher peak conductivities.

Weger and Gutfreund [33] have discussed both the pressure dependence and the constant volume temperature dependence of the resistivity for the electronlibron scattering model [8]. They consider that the Grüneison constant required to explain the high temperature behaviour is physically reasonable, but do require that at lower temperatures there should still be a $T^{2}$ law. We do not find evidence for such behaviour.

The electron-spin excitation collision model proposed by Jerome [6] has a linear temperature dependence at constant volume in one dimension $\left(t_{\perp}<k T\right.$ holds for TTF-TCNQ in the metallic regime [27]). The enhanced scattering cross-section is very sensitive to small variations in $U n\left(E_{\mathrm{F}}\right)$ when this quantity is close to unity, and the relatively small changes in $n\left(E_{\mathrm{F}}\right)$ with pressure result in the large pressure dependence that is observed.

Acknowledgments. - We thank J. R. Cooper for numerous and useful discussions and G. Malfait and A. Andrieux for their skilful assistance. We acknowledge L. Giral, J. M. Fabre and E. Aharon Shalom for the preparation of the samples used in this work. 


\section{References}

[1] Groff, R. P., Suna, A. and Merrifield, R. E., Phys. Rev. Lett. 33 (1974) 418.

[2] Ferraris, J. P. and Finnegan, T. F., Solid State Commun. 18 (1976) 1169.

[3] Cohen, M. J., Coleman, L. B., Garito, A. F. and Heeger, A. J., Phys. Rev. B 13 (1976) 5111.

[4] Thomas, G. A. et al., Phys. Rev. B 13 (1976) 5105.

[5] Seiden, P. E. and CABIB, D., Phys. Rev. B 13 (1976) 1846.

[6] Jerome, D., J. Physique Lett. 38 (1977) 489.

[7] Conwell, E. M., Phys. Rev. Lett. 39 (1977) 777.

[8] Gutfreund, H. and Weger, M., Phys. Rev. B 16 (1977) 1753.

[9] Madhukar, A. and Cohen, M. H., Phys. Rev. Lett. 38 (1977) 85.

[10] Gogolin, A. A., Vi Mel'Nikov and Rashba, E. I., Zh ETF 69 (1975) 327, and Proc. Conf. on Organic Conductors and Semi conductors, Siofok, Hungary 1976. (Springer-Verlag) Lecture Notes in Physics 65 (1977).

[11] Abrikosov, A. A. and Ryzhkin, I. A., Solid State Commun. 24 (1977) 317, 319.

[12] CoOper, J. R., Phys. Rev. B Comments.

[13] Debray, D., Millet, R., Jerome, D., Barisic, S., Giral, L. and Fabre, J. M., J. Physique Lett. 38 (1977) 227.

[14] Kistenmacher, T. J., Phillips, T. E. and Cowan, D. O., Acta Crystallogr. B 30 (1974) 763.

[15] Schultz, A. J., Stucky, G. D., Blessing, R. H. and Coppens, P., J. Am. Chem. Soc. 98 (1976) 3194.

[16] Corfield, P. and La Placa, S., unpublished, but limited results appear in

Etemad, S., Engler, A. M., Schultz, T. D., Penney, T. and Scott, B. A., Phys. Rev. B 17 (1978) 513.

[17] Greene, R. L., Mayerle, J. J., Schumaker, R., Castro, G., Chaikin, P. M., Etemad, S. and La Placa, S. J., Solid State Commun. 20 (1976) 943.
[18] Phillips, T. E., Kistenmacher, T. J., Bloch, A. N. and Cowan, D. O., J. Chem. Soc. Chem. Commun. (1976) 334.

[19] Chu, C. W., Harper, J. M. E., Geballe, T. H. and Greene, R. L., Phys. Rev. Lett. 31 (1973) 1491.

[20] Jerome, D., Muller, W. and Weger, M., J. Physique Lett. 35 (1974) 277.

[21] Cooper, J. R., Jerome, D., Weger, M. and Etemad, S., J. Physique Lett. 36 (1975) 219.

[22] Delaplace, R., Malfait, G. and Jerome, D., Rev. Phys. Appl. 11 (1976) 327.

[23] Cooper, J. R., Lukatela, J., MiljaK, M., Fabre, J. M. and Giral, L., Solid State Commun. (1978).

[24] Friend, R. H., Jerome, D., FABre, J. M., Giral, L. and BechgaArd, K., J. Phys. C 11 (1978) 263.

[25] Schafer, D. E., Wudl, F., Thomas, G. A., Ferraris, J. P. and Cowan, D. O., Solid State Commun. 14 (1974) 347.

[26] Cooper, J. R., Jerome, D., Etemad, S. and Engler, E. M., Solid State Commun. 22 (1977) 257.

[27] Soda, G., Jerome, D., Weger, M., Alizon, J., Gallice, J., Robert, H., Fabre, J. M. and Giral, L., J. Physique 38 (1977) 931.

[28] Bouffard, S. and ZupPiroli, L., Private communication.

[29] Shirane, G., Shapiro, S. M., Comes, R., Garito, A. F. and HeEger, A. J., Phys. Rev. B 14 (1976) 2325

[30] JeRICHO, M. H. (Unpublished).

[31] Schafer, D. A., Thomas, G. A. and Wudl, F., Phys. Rev. B 12 (1975) 5532.

[32] Tiedje, T., Haering, R. R., Jericho, M. H., Roger, W. A. and Simpson, A., Solid State Commun. 23 (1977) 713.

[33] Weger, M. and Gutfreund, H., Preprint. 